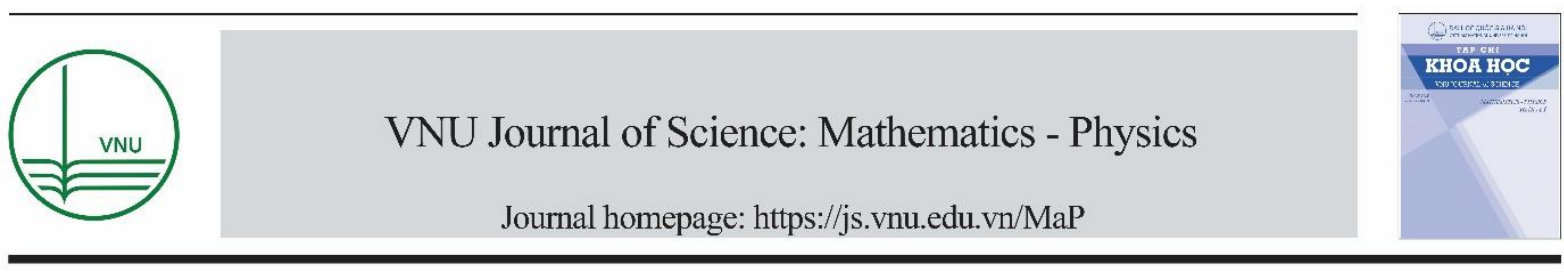

Original Article

\title{
Theoretical Prediction of the Structural and Electronic Properties of a Single Layer Graphene-like Two-dimensional Janus GaInSTe
}

\author{
Nguyen Van Chuong ${ }^{1, *}$, Nguyen Ngoc $\mathrm{Hieu}^{2}$, Nguyen Van Hieu ${ }^{3}$ \\ ${ }^{1}$ Le Quy Don Technical University, 236 Hoang Quoc Viet, Bac Tu Liem, Hanoi, Vietnam \\ ${ }^{2}$ Institute of Research and Development, Duy Tan University, 3 Quang Trung, Da Nang, Vietnam \\ ${ }^{3}$ University of Science and Education, 458 Ton Duc Thang, Lien Chieu, Da Nang, Vietnam \\ Received 04 August 2020 \\ Revised 05 November 2020; Accepted 15 December 2020
}

\begin{abstract}
This paper constructs a new type of two-dimensional graphene-like Janus GaInSTe monolayer and systematically investigates its structural and electronic properties as well as the effect of external electric field using first-principles calculations. In the ground state, Janus GaInSTe monolayer is dynamically stable with no imaginary frequencies in its phonon spectrum and possesses a direct band gap semiconductor. The band gap of Janus GaInSTe monolayer can be tuned by applying an electric field, which leads the different transitions from semiconductor to metal, and from indirect to direct band gap. These findings show a great potential application of Janus GaInSTe material for designing next-generation devices.

Keywords: Graphene, two-dimensional materials, Janus GaInSTe, first-principles calculations, electric field.
\end{abstract}

\section{Introduction}

The successful exfoliation of graphene [1] has opened a new direction in materials science, especially in nanomaterials. Owing to its extraordinary properties, graphene is considered as one of the most attractive materials. Unfortunately, the lack of a band gap in graphene [2] has limited its applications in high-speed electronic nanodevices [3]. Nowadays, there have been several strategies to

\footnotetext{
*Corresponding author.

Email address: chuong.vnguyen@1qdtu.edu.vn
}

https//doi.org/ 10.25073/2588-1124/vnumap.4408 
open a sizable band gap in graphene, such as the electric field [4], heterostructures [5, 6], and doping [7].

In parallel with finding a way to overcome this limitation of graphene, a new research direction that is looking for alternative materials has emerged strongly in the last five years. This new research has focused on 2D materials such as phosphorene [8], transition metal dichalcogenides (TMDs) [9], silicene [10]. Unlike graphene, these 2D materials are semiconductors with interesting properties and they become potential candidates for applications in nanotechnology, such as field-effect transistors (FETs) and photodetectors. These application potentials have prompted scientists to continue to study the outstanding electronic and transport properties of these materials and to explore their application potential for designing high-performance optoelectronic nanodevices. Recently, Janus structure of transition metal dichalcogenide MoSSe has been successfully synthesized by experiments [11]. The asymmetric out-of-plane geometric structure of MoSSe has been observed by means of scanning transmission electron microscopy and energy-dependent X-ray photoelectron spectroscopy [11]. It is well-known that the electronic and transport properties of the $2 \mathrm{D}$ materials are very sensitive to structural perfection and geometric symmetry plays a key role in determining their physical properties. The breaking of mirror symmetry in the Janus structures can result in many novel properties compared to the original material $\mathrm{MoS}_{2}$. The successfully experimental synthesis of MoSSe monolayer from $\mathrm{MoS}_{2}$ is an important milestone, which marks the 2D family of having a new member. Recently, Janus structures formed from the monochalcogenides, such as Janus group III monochalcogenides [12] have been theoretically investigated. Huang and co-workers demonstrated that Janus monochalcogenides $\mathrm{M}_{2} \mathrm{XY}(\mathrm{M}=\mathrm{Ga}, \mathrm{In} ; \mathrm{X}=\mathrm{S}, \mathrm{Se}, \mathrm{Te})$ can be a perfect candidate for applications in the photocatalytic performance [13]. Motivated by recent successes in studying Janus structures, the present work constructs a new graphene-like 2D Janus GaInSTe monolayer and investigates its structural and electronic properties by using first-principle calculations. The effect of the external electric field on the electronic properties of GaInSTe monolayer is also examined in this work.

\section{Computational Details}

In the present work, all density functional theory (DFT) calculations are performed in the Quantum Espresso package [14]. The structural geometry of such monolayer was fully optimized using projected augmented wave method [15] based on generalized gradient approximation (GGA) [16] within Perdew, Burke, and Ernzerhof (PBE). The Heyd-Scuseria-Ernzerhof hybrid functional (HSE06) was used to obtain more accurate band gap of materials [17]. The cut-off energy of $500 \mathrm{eV}$ was tested and used for calculating the electronic structure. The Brillouin zone (BZ) integration of $6 \times 6 \times 1 \mathrm{k}$-mesh was sampled for all the calculations. To avoid the interaction between the adjacent sublayers, a large vaccum thickness of $30 \AA$ was set. The total energy was converged when the force and energy were less than $0.01 \mathrm{eV} \times \AA^{-1}$ and $10^{-5} \mathrm{eV}$, respectively. Phonon dispersion calculations were calculated using PHONOPHY code [18].

\section{Results and Discussion}

The crystal structure of Janus GaInSTe monolayer is depicted in Figure 1, which contains four sublayers that stacked in the sequence $\mathrm{S}-\mathrm{Ga}-\mathrm{In}-\mathrm{Te}$. This Janus structure could be generated in two ways: in the first pattern, we replace one of two sublayers of Ga by Indium (In) atom in parent $\mathrm{GaS}$ monolayer, resulting in the formation of Janus $\mathrm{GaInS}_{2}$ monolayer; in the second pattern, the inner two layers of $\mathrm{Ga}$ and $\mathrm{In}$ atoms remain unchanged, only $\mathrm{S}$ atom of the top layer of $\mathrm{GaInS}_{2}$ is replaced by Te 
atom to give rise to the formation of the Janus GaInSTe monolayer, as depicted in Figure 1. After geometric optimization, the obtained lattice parameter of the Janus GaInSTe monolayer is calculated to be $4.03 \AA$, which is larger than that of the parent GaS monolayer of $3.585 \AA$ [19]. The calculated bond lengths of Ga-In, Ga-S, In-Te are $2.64 \AA$, $2.49 \AA$, and $2.80 \AA$, respectively, whereas the vertical S-Te thickness is calculated to be $5.13 \AA$, which is larger than that in the parent GaS monolayer.

(a)

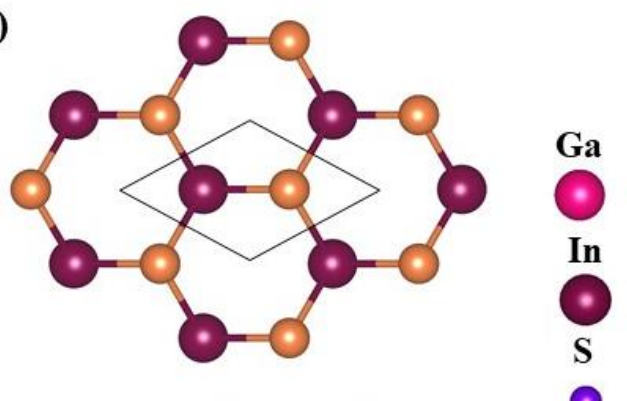

(b)

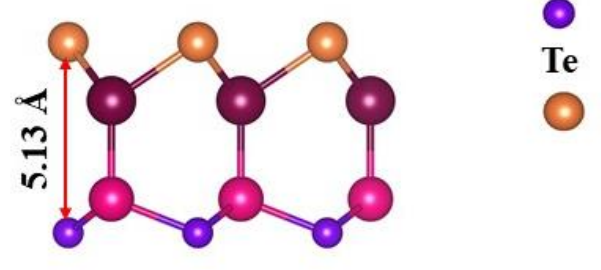

Figure 1. (a) Top view and (b) Side view of the atomic structure of a single layer graphene-like Janus GaInSTe, respectively.

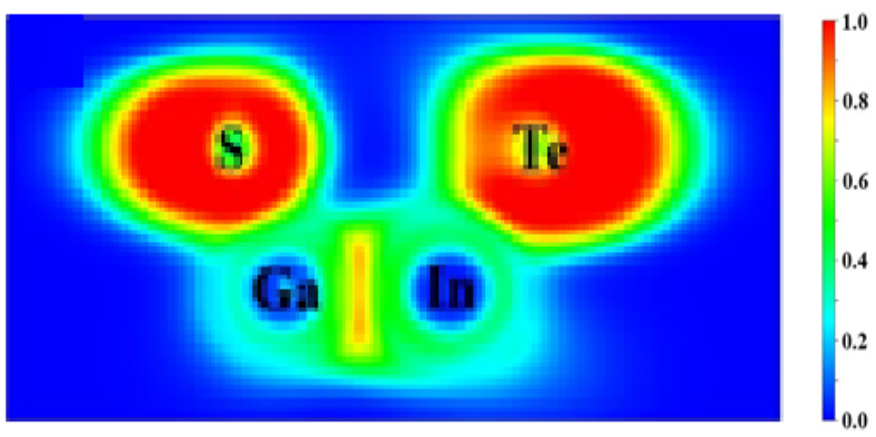

Figure 2. Electron contour map of single-layer Janus GaInSTe.

To have a better understanding of the covalent bonding in Janus GaInSTe monolayer, we further plot the electron contour map of Janus GaInSTe monolayer, as illustrated in Figure 2. We can find that the electron contour map of such material can be described in real space. We set the isosurface from 0 to 1 . The " 0 " value, i.e. blue regions represent the delocalized electron, while the " 1 " value, i.e. red regions stand for the localized electron. One can find that the red regions are mainly visualized by the $\mathrm{S}$ and Te atoms, while blue regions are located around the $\mathrm{Ga}$ and $\mathrm{In}$ atoms. This finding means that the electron states in the $\mathrm{S}$ and Se directions are localized. The green regions are mainly visualized in the $\mathrm{Ga}(\mathrm{In})-\mathrm{S}(\mathrm{Se})$ bonding, thus demonstrating that these bondings are characterized by the covalent bonding. 

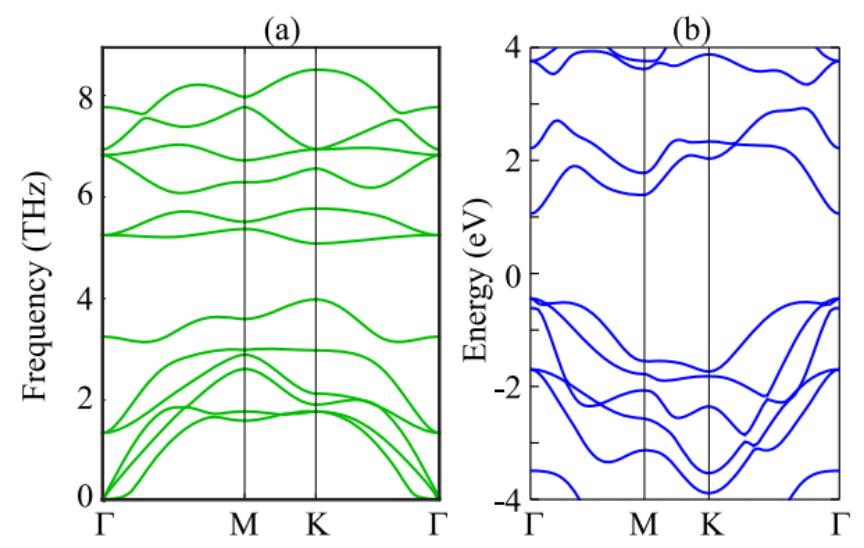

Figure 3. (a) Phonon dispersion curves and (b) Band structure of a graphene-like 2D Janus GaInSTe.

In order to check the dynamical stability of a single layer Janus GaInSTe, we further calculate its phonon dispersion curves, as depicted in Figure 3 (a). One can find that the Janus GaInSTe monolayer has no imaginary frequencies in the phonon spectrum, indicating its dynamical stability. The electronic band structure of Janus GaInSTe monolayer is also calculated and displayed in Figure 3 (b). The Janus GaInSTe monolayer exhibits a semiconducting nature with a direct band gap. The band gap of Janus GaInSTe monolayer calculated by PBE and HSE06 is $0.80 \mathrm{eV}$ and $1.50 \mathrm{eV}$, respectively. The values are smaller than those of GaS monolayer of $2.37 \mathrm{eV}$ and $3.24 \mathrm{eV}$ by PBE and HSE06 calculations, respectively. It should be noted that the traditional PBE functional always underestimates the band gap of materials. While the hybrid functional HSE06 can be used to acquire an accurate band gap. Furthermore, both the valence band maximum (VBM) and conduction band minimum (CBM) are located right at the $\Gamma$ point. To have a deep insight into the band structure, we further calculate the weighted band structure of Janus GaInSTe monolayer, as shown in Figure 4. Our calculations demonstrate that the VBM is mainly contributed by Te-p state. While the CBM of GaInSTe is contributed by $\mathrm{S}-s$ state.

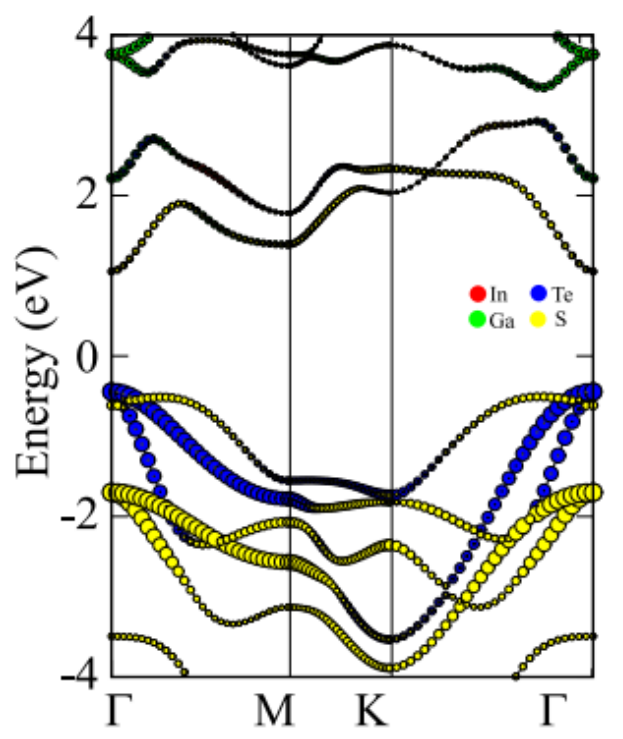

Figure 4. The weighted band structure of all atoms in Janus GaInSTe. 

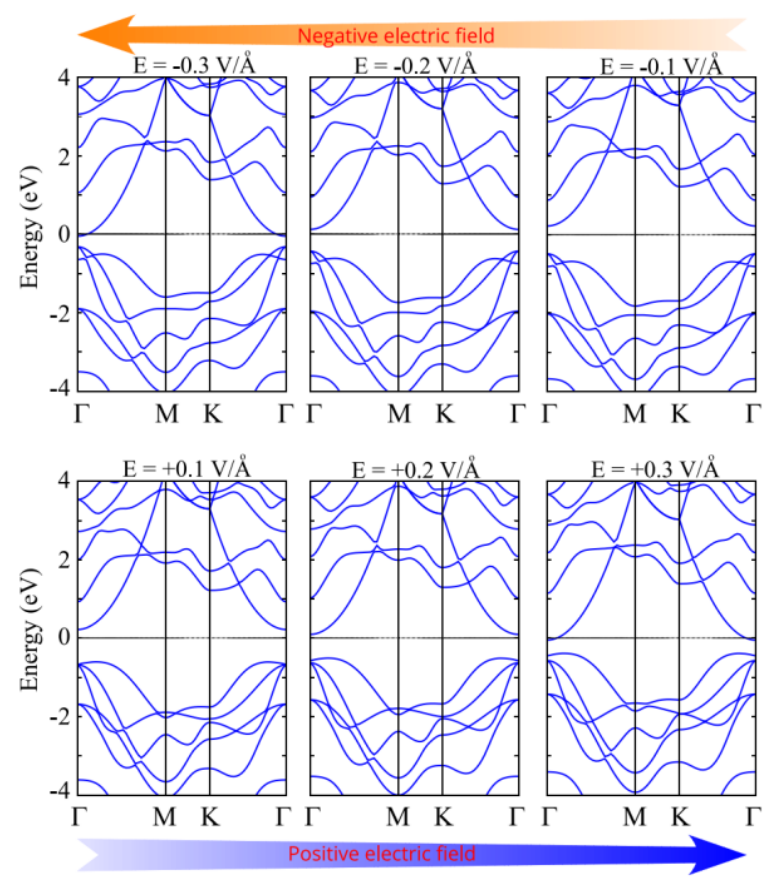

Figure 5. Band structures of GaInSTe under different strengths of negative and positive electric fields.

What is more, the performances of electronic devices depend mainly on the properties of the material, which can be adjusted by several external conditions, such as strain engineering, doping, and external electric field. Recently, the external electric field is devoted to being one of the most common strategies for modifying the electronic properties of materials. Therefore, we further consider the effect of the external electric field on electronic properties of Janus GaInSTe monolayer. The strength of the electric field is ranging from $-0.3 \mathrm{~V} / \AA$ to $+0.3 \mathrm{~V} / \AA$. The positive direction of the electric field points from the bottom to the top of the Janus monolayer along the $z$-direction. As above mentioned, in the ground state, Janus GaInSTe monolayer possesses a direct band gap semiconductor with both the CBM and VBM at the $\Gamma$ point. Interestingly, the band structure of GaInSTe is greatly affected by the external field, as depicted in Figure 5. The different transitions from semiconductor to metal, and direct to indirect band gap are observed under electric filed. The indirect band gap at $\Gamma-\mathrm{M}$ point is achieved by applying an electric field of $+0.1 \mathrm{~V} / \AA$. Furthermore, the metallic nature is observed when it is subjected to an electric field of +0.3 , and $-0.3 \mathrm{~V} / \AA$.

\section{Conclusion}

In summary, we have systematically investigated the structural and electronic properties of a new graphene-like 2D Janus GaInSTe monolayer as well as the effect of external electric field using firstprinciples calculations. Janus GaInSTe monolayer is dynamically stable and possesses a direct band gap semiconductor. The band structure of GaInSTe is greatly affected by the external field. The different transitions from semiconductor to metal, and indirect to direct band gap are observed under electric gating. Our calculated results contribute to an insightful view of the electronic properties of graphene-like Janus GaInSTe material. 


\section{Acknowledgments}

This research is funded by Vietnam National Foundation for Science and Technology Development (NAFOSTED) under grant No. 103.01-2019.05.

\section{References}

[1] K. S. Novoselov, A. K. Geim, S. V. Morozov, D. Jiang, Y. Zhang, S. V. Dubonos, I. V. Grigorieva, A. A. Firsov, Electric Field Effect in Atomically Thin Carbon Films, Science, Vol. 306, No. 5696, 2004, pp. 666-669, https://doi.org/10.1126/science.1102896.

[2] A. K. Geim, K. S. Novoselov, The Rise of Graphene, Nat. Mater., Vol. 6, No. 3, 2007, pp. 183-191, https://doi.org/10.1038/nmat1849.

[3] M. Jang, H. Kim, Y. Son, H. Atwater, W. Goddard, Graphene Field Effect Transistor without an Energy Gap, Proc. Natl. Acad. Sci., Vol. 110, No. 22, 2013, pp. 8786-8789, https://doi.org/10.1073/pnas.1305416110.

[4] A. Avetisyan, B. Partoens, F. M. Peeters, Electric Field Tuning of the Band Gap in Graphene Multilayers, Phys. Rev. B, Vol. 79, No. 3, 2009, pp. 035421-035627, https://doi.org/10.1103/PhysRevB.79.035421.

[5] C. V. Nguyen, M. Idrees, H. V. Phuc, N. N. Hieu, N. T. T. Binh, B. Amin, T. V. Vu, Interlayer Coupling and Electric Field Controllable Schottky Barriers and Contact Types in Graphene/ $\mathrm{PbI}_{2}$ Heterostructures, Phys. Rev. B, Vol. 101, No. 23, 2020, pp. 235419-235426, https://doi.org/10.1103/PhysRevB.101.235419.

[6] T. V. Vu, N. N. Hieu, H. V. Phuc, N. V. Hieu, H. D. Bui, M. Idrees, B. Amin, C. V. Nguyen, Graphene/WSeTe van der Waals Heterostructure: Controllable Electronic Properties and Schottky Barrier via Interlayer Coupling and Electric Field, Appl. Surf. Sci., Vol. 507, 2020, pp. 145036-145045, https://doi.org/10.1016/j.apsusc.2019.145036.

[7] A. Hussain, S. Ullah, M. A. Farhan, Fine Tuning the Band-Gap of Graphene by Atomic and Molecular Doping: A Density Functional Theory Study, RSC Adv., Vol. 6, No. 61, 2016, pp. 55990-56003, https://doi.org/10.1039/C6RA04782C.

[8] H. Liu, A. T. Neal, Z. Zhu, Z. Luo, Z. Xu, D. Tomanek, P. D. Ye, Phosphorene: An Unexplored 2D Semiconductor with a High Hole Mobility, ACS Nano, Vol. 8, No. 4, 2014, pp. 4033-4041, https://doi.org/10.1021/nn501226z.

[9] S. Manzeli, D. Ovchinnikov, D. Pasquier, O. V. Yazyev, A. Kis, 2D Transition Metal Dichalcogenides, Nat. Rev. Mater., Vol. 2, No. 8, 2017, pp. 17033-17047. https://doi.org/10.1038/natrevmats.2017.33.

[10] P. Vogt, P. D. Padova, C. Quaresima, J. Avila, E. Frantzeskakis, M. C. Asensio, A. Resta, B. Ealet, G. L. Lay, Silicene: Compelling Experimental Evidence for Graphenelike Two-Dimensional Silicon, Phys. Rev. Lett., Vol. 108, No. 15, 2012, pp. 155501-155505, https://doi.org/10.1103/PhysRevLett.108.155501.

[11] A. Lu, H. Zhu, J. Xiao, C. Chuu, Y. Han, M. Chiu, C. Cheng, C. Yang, K. Wei, Y. Yang, Y. Wang, D. Sokaras, D. Nordlund, P. Yang, D. A. Muller, M. Y. Chou, X. Zhang, L. J. Li, Janus Monolayers of Transition Metal Dichalcogenides, Nat. Nanotechnol., Vol. 12, No. 8, 2017, pp. 744-749, https://doi.org/10.1038/nnano.2017.100.

[12] W. Wan, S. Zhao, Y. Ge, Y. Liu, Phonon and Electron Transport in Janus Monolayers based on InSe, J. Phys. Condens. Matter, Vol. 31, No. 43, 2019, pp. 435501-435511, https://doi.org/10.1088/1361-648X/ab2e7d.

[13] A. Huang, W. Shi, Z. Wang, Optical Properties and Photocatalytic Applications of Two-Dimensional Janus Group-III Monochalcogenides, J. Phys. Chem. C, Vol. 123, No. 18, 2019, pp. 11388-11396, https://doi.org/10.1021/acs.jpcc.8b12450.

[14] P. Giannozzi, S. Baroni, N. Bonini, M. Calandra, R. Car, C. Cavazzoni, D. Ceresoli, G. L. Chiarotti, M. Cococcioni, I. Dabo, A. D. Corso, S. D. Gironcoli, S. Fabris, G. Fratesi, R. Gebauer, U. Gerstmann, C. Gougoussis, A. Kokalj, M. Lazzeri, L.M. Samos, N. Marzari, F. Mauri, R. Mazzarello, S. Paolini, A. Pasquarello, L. Paulatto, C. Sbraccia, S. Scandolo, G. Sclauzero, A. P. Seitsonen, A. Smogunov, P. Umari, R. M. Wentzcovitch, QUANTUM ESPRESSO: A Modular and Open-Source Software Project for Quantum Simulations of Materials, J. Phys. Condens. Matter, Vol. 21, No. 39, 2009, pp. 395502-395521, https://doi.org/10.1088/0953-8984/21/39/395502.

[15] P. E. Blöchl, Projector Augmented-Wave Method, Phys. Rev. B, Vol. 50, No. 24, 1994, pp. 17953-17979, https://doi.org/10.1103/PhysRevB.50.17953. 
[16] J. P. Perdew, K. Burke, M. Ernzerhof, Generalized Gradient Approximation Made Simple, Phys. Rev. Lett., Vol. 77, No. 18, 1996, pp. 3865-3868, https://doi.org/10.1103/PhysRevLett.77.3865.

[17] J. Heyd, G. E. Scuseria, Efficient Hybrid Density Functional Calculations in Solids: Assessment of the HeydScuseria-Ernzerhof Screened Coulomb Hybrid Functional, J. Chem. Phys., Vol. 121, No. 3, 2004, pp. 11871192, https://doi.org/10.1063/1.1760074.

[18] A. Carrerasa, A. Togo, I. Tanaka, Dynaphopy: A Code for Extracting Phonon Quasiparticles from Molecular Dynamics Simulations, Comput. Phys. Commun., Vol. 221, 2017, pp. 221-234, https://doi.org/10.1016/j.cpc.2017.08.017.

[19] K. D. Pham, N. N. Hieu, H. V. Phuc, I. A. Fedorov, C. A. Duque, B. Amin, C. V. Nguyen, Layered Graphene/GaS van der Waals Heterostructure: Controlling the Electronic Properties and Schottky Barrier by Vertical Strain, Appl. Phys. Lett., Vol. 113, No. 17, 2018, pp. 171605-171609, https://doi.org/10.1063/1.5055616. 